\title{
2015 Cole Prize in Algebra
}

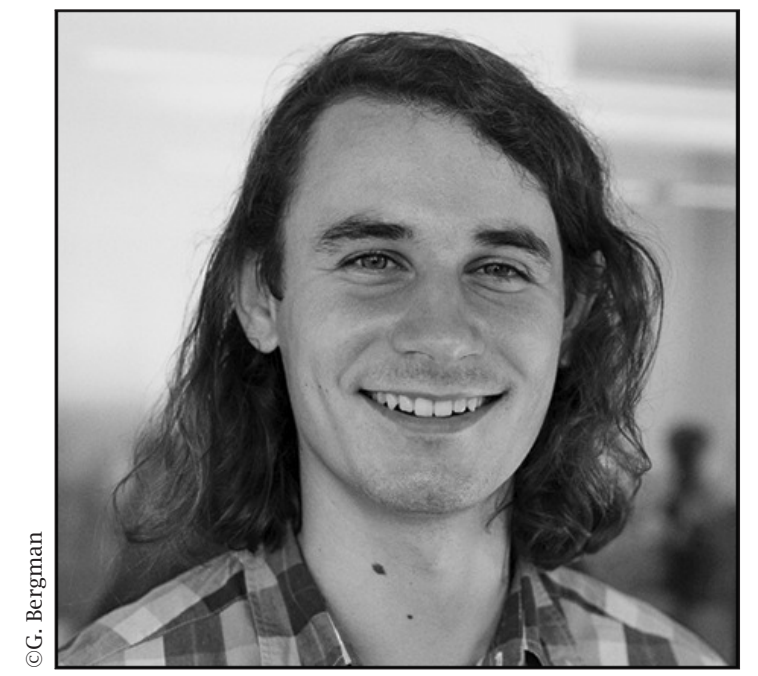

Peter Scholze

Peter Scholze was awarded the 2015 Cole Prize in Algebra at the 121st Annual Meeting of the AMS in San Antonio, Texas, in January 2015.

\section{Citation}

The 2015 Frank Nelson Cole Prize in Algebra is awarded to Peter Scholze for his work on perfectoid spaces which has led to a solution of an important special case of the weight-monodromy conjecture of Deligne ("Perfectoid spaces", Publ. Math. Inst. Hautes Études Sci. 116 (2012), no. 1, 245-313). Scholze has also found other spectacular applications of perfectoid spaces to $p$-adic Hodge theory and the $p$-adic geometry of Shimura varieties (and many more are expected).

A perfectoid field $K$ is a complete nonArchimedean field $K$ with characteristic $p>0$ residue field and a nondiscrete valuation of rank 1 such that the Frobenius map $\Phi: \mathcal{O}_{K} / p \rightarrow \mathcal{O}_{K} / p$ is surjective, where $\mathcal{O}_{K} \subset K$ is the subring of elements of norm $\leq 1$. To any perfectoid field $K$, one can associate a second perfectoid field $K^{b}$ of characteristic $p$ given by the fraction field of

$$
\left\{\left(a_{n}\right)_{n \in \mathbf{N}} \in\left(\mathcal{O}_{K} / p\right)^{\mathbf{N}} ; a_{n+1}^{p}=a_{n} \quad \forall n\right\} .
$$

DOI: http://dx.doi.org/10.1090/noti1227
For example, if $K$ is the completion of $\mathbb{Q}_{p}\left(p^{1 / p^{\infty}}\right)$, then $K^{b}$ is the completion of $\mathbb{F}_{p}((t))\left(t^{1 / p^{\infty}}\right)$, and in this case, by a theorem of Fontaine and Wintenberger, there is a canonical isomorphism of absolute Galois groups $\operatorname{Gal}(\bar{K} / K) \cong \operatorname{Gal}\left(\bar{K}^{b} / K^{b}\right)$. In his work on perfectoid spaces, Scholze generalizes this result obtaining a comparison between geometric objects over $K$ and geometric objects over $K^{b}$. More precisely, he defines the notion of perfectoid space over $K$ (a kind of analytic space over $K$ ), and he shows that the category of perfectoid spaces over $K$ and that of perfectoid spaces over $K^{b}$ are equivalent. This provides a link between geometry over local fields of mixed characteristic and geometry over local fields of equal characteristic, where the questions are usually more tractable. Using the theory of perfectoid spaces, Scholze shows that Deligne's weight-monodromy conjecture over $p$-adic fields will follow from the known equal characteristic case if one can show that certain fractal-like subsets of $\mathbb{P}_{K^{b}}^{n}$ can be approximated by algebraic subvarieties. He also shows that smooth complete intersection subschemes of toric varieties satisfy this approximation result, and hence Deligne's weight-monodromy conjecture holds for these varieties.

\section{Biographical Sketch}

Peter Scholze was born in Dresden, Germany, in 1987. After going to high school at the HeinrichHertz-Oberschule in Berlin, he studied in Bonn, where he earned his $\mathrm{PhD}$ in 2012 under Michael Rapoport. His thesis was on perfectoid spaces and the weight-monodromy conjecture. In July 2011 he was appointed as a Clay Research Fellow for five years, and in 2012 he obtained a Hausdorff Chair at the University of Bonn. In 2013 he received the Prix Peccot of the Collège de France and the SASTRA Ramanujan Prize. Moreover, he received the Clay Research Award and was an invited speaker at the ICM 2014.

\section{Response from Peter Scholze}

It is a great honor to receive the 2015 Frank Nelson Cole Prize in Algebra. Looking back at the list of previous recipients of the prize, I am humbled to be named on this list. I am indebted to my advisor 
Michael Rapoport, who not only suggested that I should work on the weight-monodromy conjecture but was also convinced that it should be possible to reduce it to its equal characteristic version after a very ramified base change. Moreover, I would like to thank Gerd Faltings for the courses he taught at the University of Bonn. Although I felt like I never understood anything in his lectures, I still think that I learned an enormous amount and am heavily influenced by them. Finally, I want to thank my wife, daughter, parents, and friends for everything they have done for me.

\section{About the Prize}

The Cole Prize in Algebra is awarded every three years for a notable research memoir in algebra that has appeared during the previous six years. The awarding of this prize alternates with the awarding of the Cole Prize in Number Theory, also given every three years. These prizes were established in 1928 to honor Frank Nelson Cole (1861-1926) on the occasion of his retirement as secretary of the AMS after twenty-five years of service. He also served as editor-in-chief of the Bulletin for twentyone years. The Cole Prize carries a cash award of US $\$ 5,000$. The Cole Prize in Algebra is awarded by the AMS Council acting on the recommendation of a selection committee. For 2015 the members of the selection committee were Christopher D. Hacon, George Lusztig (chair), and Karen E. Smith.

Previous recipients of the Cole Prize in Algebra are: L. E. Dickson (1928), A. Adrian Albert (1939), Oscar Zariski (1944), Richard Brauer (1949), Harish-Chandra (1954), Serge Lang (1960), Maxwell A. Rosenlicht (1960), Walter Feit and John G. Thompson (1965), John R. Stallings (1970), Richard G. Swan (1970), Hyman Bass (1975), Daniel G. Quillen (1975), Michael Aschbacher (1980), Melvin Hochster (1980), George Lusztig (1985), Shigefumi Mori (1990), Michel Raynaud and David Harbater (1995), Andrei Suslin (2000), Aise Johan de Jong (2000), Hiraku Nakajima (2003), János Kollár (2006), Christopher Hacon and James McKernan (2009), and Alexander S. Merkurjev (2012).

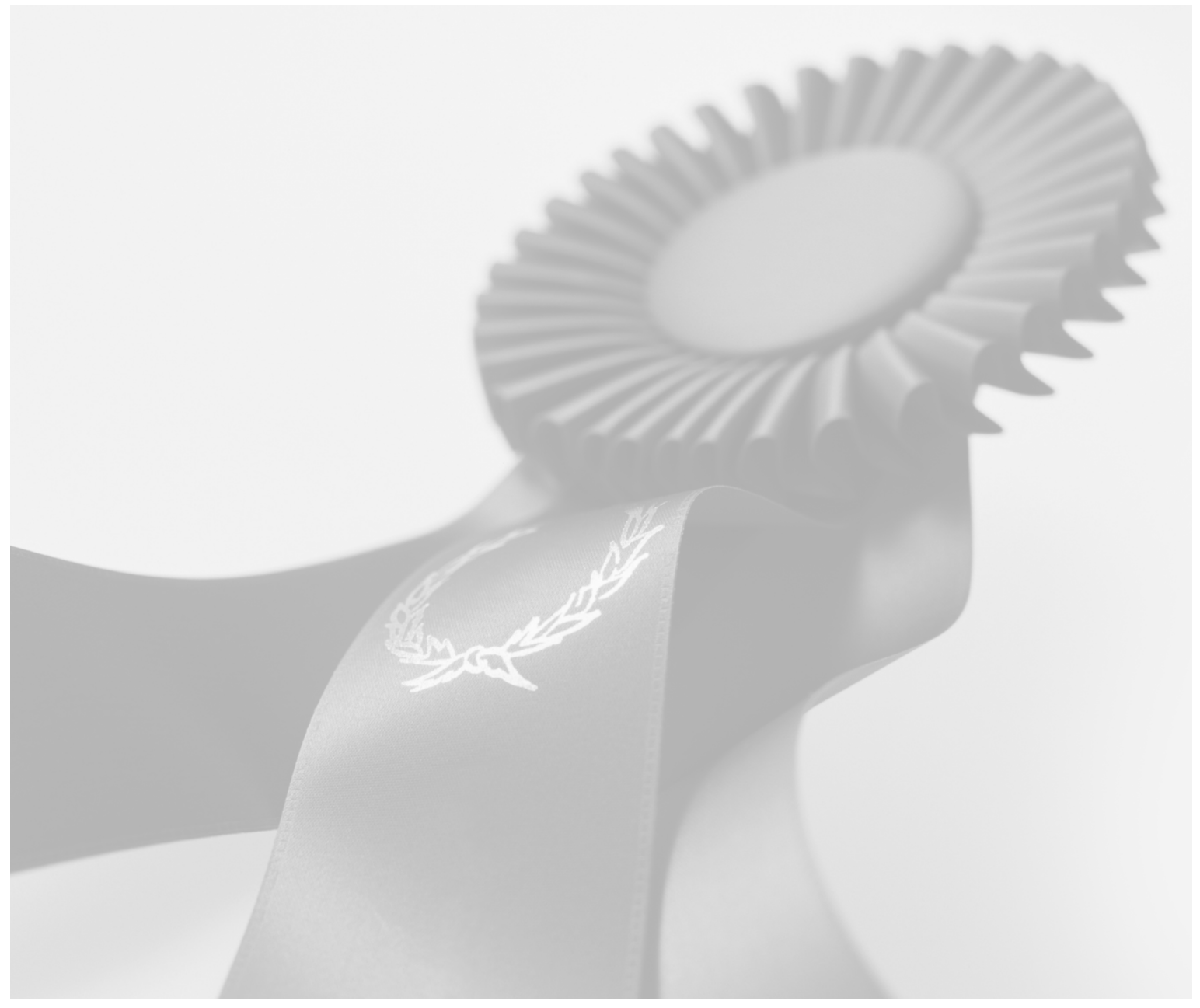

